\title{
Functional Topography and Development of Inhibitory Reticulothalamic Barreloid Projections
}

\author{
Kazuo Imaizumi ${ }^{1,2,3 *}$, Yuchio Yanagawa ${ }^{4}$, Guoping Feng ${ }^{2,3}$ and Charles C. Lee ${ }^{1}$ \\ ${ }^{1}$ Department of Comparative Biomedical Sciences, Louisiana State University, School of Veterinary Medicine, Baton Rouge, \\ LA, United States, ${ }^{2}$ McGovern Institute for Brain Research, Department of Brain and Cognitive Sciences, Massachusetts \\ Institute of Technology, Cambridge, MA, United States, ${ }^{3}$ Stanley Center for Psychiatric Research, Broad Institute of MIT and \\ Harvard, Cambridge, MA, United States, ${ }^{4}$ Department of Genetic and Behavioral Neuroscience, Gunma University, \\ Graduate School of Medicine, Maebashi, Japan
}

\section{OPEN ACCESS}

Edited by:

Christiaan P. J. De Kock, VU University Amsterdam,

Netherlands

Reviewed by: Carlos Avendaño, Universidad Autónoma de Madrid,

Spain

Joshua C. Brumberg,

Queens College (CUNY), United States

Rajeevan Therpurakal Narayanan, Center of Advanced European Studies and Research (CAESAR),

Germany

*Correspondence: Kazuo Imaizumi

kazuo.imaizumi@wyss.harvard.edu

Received: 23 August 2018 Accepted: 08 October 2018 Published: 31 October 2018

Citation:

Imaizumi $K$, Yanagawa Y, Feng G and Lee CC (2018) Functional Topography and Development of Inhibitory Reticulothalamic Barreloid Projections.

Front. Neuroanat. 12:87. doi: 10.3389/fnana.2018.00087
The thalamic reticular nucleus (TRN) is the main source of inhibition to the somatosensory thalamus (ventrobasal nucleus, VB) in mice. However, the functional topography and development of these projections with respect to the VB barreloids has been largely unexplored. In this respect, to assist in the study of these projections, we have utilized a vesicular gamma-aminobutryic acid (GABA) transporter (VGAT)-Venus transgenic mouse line to develop a brain slice preparation that enables the rapid identification of inhibitory neurons and projections. We demonstrate the utility of our in vitro brain slice preparation for physiologically mapping inhibitory reticulothalamic (RT) topography, using laser-scanning photostimulation via glutamate uncaging. Furthermore, we utilized this slice preparation to compare the development of excitatory and inhibitory projections to VB. We found that excitatory projections to the barreloids, created by ascending projections from the brain stem, develop by postnatal day 2-3 (P2-P3). By contrast, inhibitory projections to the barreloids lag $\sim 5$ days behind excitatory projections to the barreloids, developing by P7-P8. We probed this lag in inhibitory projection development through early postnatal whisker lesions. We found that in whisker-lesioned animals, the development of inhibitory projections to the barreloids closed by P4, in register with that of the excitatory projections to the barreloids. Our findings demonstrate both developmental and topographic organizational features of the RT projection to the VB barreloids, whose mechanisms can now be further examined utilizing the VGAT-Venus mouse slice preparation that we have characterized.

Keywords: VGAT, critical period, somatosensory, thalamus, laser-scanning photostimulation

\section{INTRODUCTION}

The thalamus represents an ideal structure to assess aspects of developmental plasticity of both excitatory and inhibitory projection systems. The thalamus is the obligate neural structure conveying sensory information to the cortex (Sherman and Guillery, 2002; Jones, 2007; Erzurumlu and Gaspar, 2012; Imaizumi and Lee, 2014) and receives feedforward excitatory projections from subthalamic structures and feedback excitatory corticothalamic $(\mathrm{CT})$ projections from neurons in 


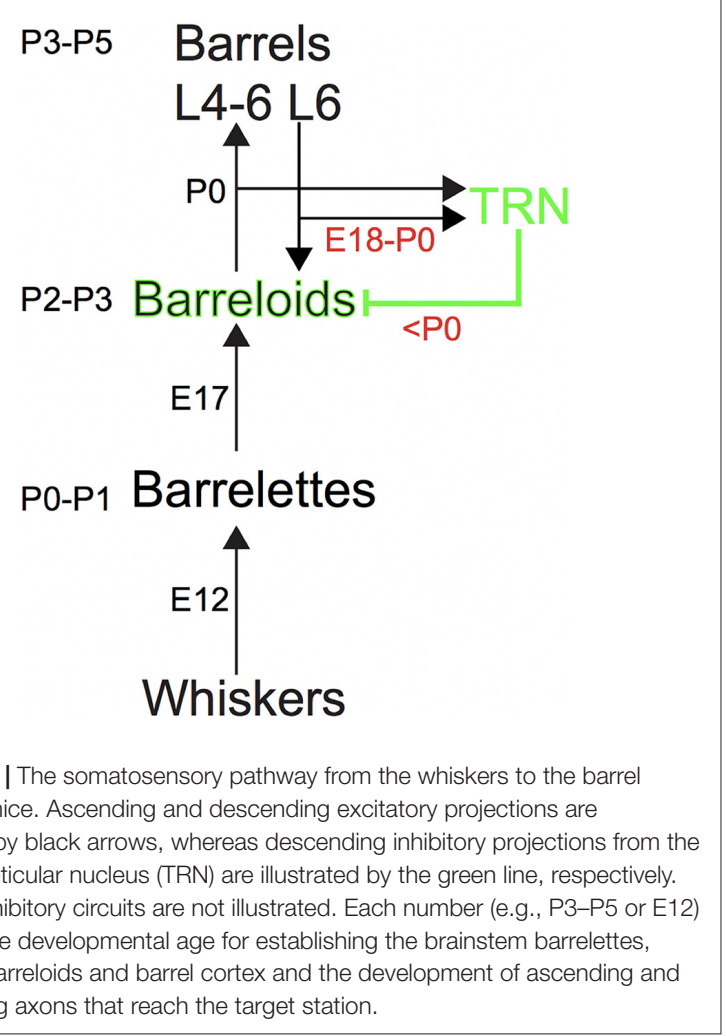

layer 6 of the neocortex (Sherman, 2016; Figure 1). In addition, the thalamus receives feedback inhibitory reticulothalamic (RT) projections from the thalamic reticular nucleus (TRN), which along with the zona incerta, are sources of inhibition to the ventrobasal nucleus (VB) in mice that lack local VB interneurons (Guillery and Harting, 2003; Pinault, 2004; Lam and Sherman, 2005; Sherman, 2016; Figure 1). Thus, the thalamus integrates excitatory and inhibitory projections in a thalamo-corticothalamic loop.

Despite its functional importance, structural plasticity in the thalamus has been much less appreciated than in the neocortex. Moreover, most ascending projections from the brainstem and thalamocortical (TC) projections are excitatory, such that studies of developmental plasticity in the thalamus have focused similarly on those excitatory projections. Interestingly, local inhibitory synapses can also be altered with excitatory synapses in developmental plasticity of the neocortex (Froemke, 2015). However, it remains unclear whether long-range inhibitory projections are similarly altered.

Even the seemingly rigid topographic maps of sensory space are amenable to structural plasticity in the developing nervous system (Sur and Leamey, 2001; Winer et al., 2004; Schreiner and Winer, 2007; Espinosa and Stryker, 2012). The well-defined whisker barrel maps in the primary somatosensory barrel cortex have demonstrated experience-dependent structural plasticity of developing TC projections (Inan and Crair, 2007; Lokmane and Garel, 2014). The mechanisms underlying such remodeling of excitatory projections involve the activitydependent strengthening and pruning of excitatory synapses.
By comparison, similar structural plasticity of inhibitory neural projections in the thalamus has not been intensively investigated, in part due to the lack of amenable preparations for investigating such changes.

Here, we take advantage of a vesicular gamma-aminobutryic acid (GABA) transporter (VGAT)-Venus transgenic mouse line to examine the developmental organization of inhibitory projections from the TRN to the barreloid field in the somatosensory thalamus (the VB nucleus). We demonstrate the utility of this preparation for in vitro slice physiological recordings to map the topographic organization of inhibitory projections to thalamic barreloids identified online. In addition, we demonstrate the development and structural plasticity of inhibitory projections to the barreloids using this preparation. Overall, we demonstrate a new preparation for studying the organization, development and structural plasticity of inhibitory projections to the barreloid region of the somatosensory thalamus.

\section{MATERIALS AND METHODS}

\section{Slice Preparation}

All experimental procedures were approved by the Institutional Animal Care and Use Committee (IACUC) of the Louisiana State University School of Veterinary Medicine and the Committee on Animal Care of the Massachusetts Institute of Technology. Live slices were prepared from VGAT-Venus transgenic mice at $\mathrm{P} 11-\mathrm{P} 13$. These transgenic mice express the Venus fluorescent protein (pCS2-Venus developed in the laboratory of Dr. Atsushi Miyawaki at RIKEN, Wako, Japan) in VGAT-positive neurons (mouse line developed and shared by Dr. Yuchio Yanagawa at Gunma University) and obtained from Dr. Janice R. Naegele at Wesleyan University (Nagai et al., 2002; Wang et al., 2009).

Animals were deeply anesthetized under isoflurane. After decapitation, the brains were quickly removed and submerged in ice cold, oxygenated, artificial cerebral spinal fluid (ACSF; $125 \mathrm{mM} \mathrm{NaCl}, 3 \mathrm{mM} \mathrm{KCl}, 25 \mathrm{mM} \mathrm{NaHCO} 3,1.25 \mathrm{mM}$ $\mathrm{NaH}_{2} \mathrm{PO}_{4}, 1 \mathrm{mM} \mathrm{MgCl}, 2 \mathrm{mM} \mathrm{CaCl}, 25 \mathrm{mM}$ d-glucose). Brains were blocked to preserve the thalamic barreloids (Figure 2). The blocking cuts were similar to the blocking angles for the auditory TC slice (Cruikshank et al., 2002; Lee and Sherman, 2008). A key distinction with our preparation to that previously described for the auditory TC slice is an initial $30^{\circ}$ dorsoventral coronal blocking cut, followed by the $15^{\circ}$ semi-horizontal blocking cut, which was found to well preserve the barreloid architecture (Figure 2). The blocked brains were glued on a stage with instant glue adhesive, ethyl cyanoacrylate (Elmers Krazy Glue, High Point, NC, USA), and then $500 \mu \mathrm{m}$ thick sections were collected in ice-cold, oxygenated ACSF or sucrose-rich brain slice solution using a vibratome (World Precision Instruments, Sarasota, FL, USA; Lee et al., 2013). Collected slices were transferred to a holding chamber for $1 \mathrm{~h}$ at $32^{\circ} \mathrm{C}$ in oxygen-saturated ACSF and moved to a recording chamber perfused with oxygen-saturated aCSF at $32^{\circ} \mathrm{C}$ on a microscope stage (Siskiyou, Grants Pass, OR, USA). 


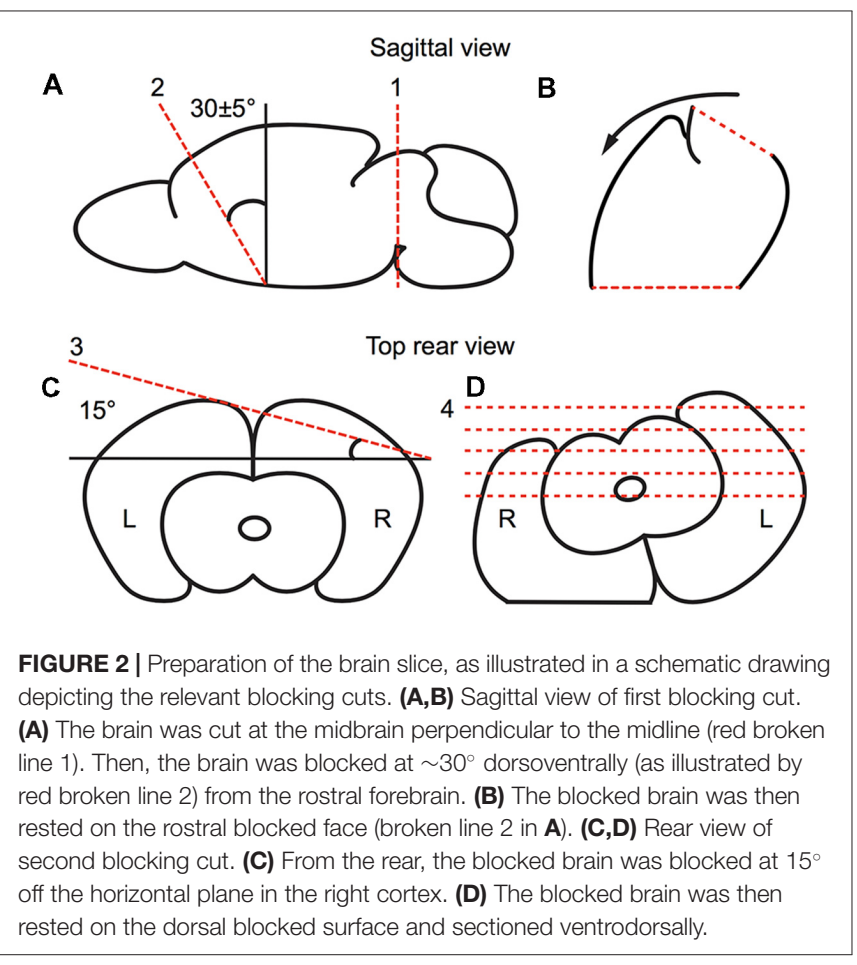

\section{Slice Physiological Recordings and Laser-Scanning Photostimulation}

Inhibitory projections from the TRN to the VB barreloids in the live slice preparation were imaged with a Retiga-EX camera (QImaging, Surrey, BC, Canada), using StreamPix5 (Norpix, Montreal, QC, Canada), mounted on an Olympus BX-51 upright microscope (Olympus, Tokyo, Japan) through a Chroma filter (41026; HQ495-30×, HQ 545-50 m, Q516 LP; Chroma, Rockingham, VT, USA; Lee and Imaizumi, 2013). Barreloids were identified and targeted on-line (Van Der Loos, 1976). Recordings were made from VB neurons at P11-13, when intrinsic membrane properties were relatively constant. To assist in isolating inhibitory currents, whole-cell recordings were made in voltage clamp mode using recording pipettes with tip resistances of 4-8 $\mathrm{M} \Omega$ filled with a cesium intracellular solution (110 mM d-gluconic acid, $110 \mathrm{mM} \mathrm{CsOH}, 10 \mathrm{mM} \mathrm{CsCl}_{2}, 1 \mathrm{mM}$ $\mathrm{CaCl}_{2}, 1$ mM EGTA, 1 mM Mg-ATP, 10 mM HEPES, $\mathrm{pH}$ 7.3) to hold the cell at $0 \mathrm{mV}$.

Uncaging of glutamate by laser-scanning photostimulation was used to identify synaptic input locations in the TRN eliciting inhibitory postsynaptic currents (IPSCs) in the recorded barreloid cells. After patching, a recirculating ACSF bath containing $0.37 \mathrm{mM}$ nitroindolinyl (NI)-caged glutamate (Sigma-Aldrich, St. Louis, MO, USA) was switched in place of the regular ACSF bath. Photolysis of the caged glutamate was made focally with a pulsed UV laser (DPSS Lasers Inc., Santa Clara, CA, USA). We used an $8 \times 16$ stimulation array with $80 \mu \mathrm{m}$ spacing between stimulation spots. We repeated the mapping procedures 3-5 times for each neuron and averaged the resultant maps using the mapAnalysis and mapAverager programs in Ephus (Janelia Farms, Jupiter, FL,
USA; Suter et al., 2010). Detailed procedures were described elsewhere (Lee and Imaizumi, 2013).

\section{Imaging VGAT-Venus in Barreloids in Fixed Tissue}

After deep anesthesia under isoflurane, brains from juvenile VGAT-Venus mice (at P4-P14) were removed and blocked as described above (Figure 2; Cruikshank et al., 2002; Lee and Sherman, 2008), and fixed by submerging in $4 \%$ paraformaldehyde (PFA, Electron Microscopy Sciences, Hatfield, $\mathrm{PA}, \mathrm{USA}$ ) in $10 \mathrm{mM}$ phosphate buffered saline (PBS, $\mathrm{pH}$ 7.3) for a few days (Lee et al., 2013). For cryoprotection, the brains were kept in $30 \%$ sucrose for 2-4 days. The blocked brains were mounted on OCT compound (Sakura Finetek, Tokyo, Japan) and sectioned at $-20^{\circ} \mathrm{C}$ using a Leica cryostat (Leica Microsystems, Buffalo Grove, IL, USA). Sections (50 $\mu \mathrm{m}$ ) were collected in $10 \mathrm{mM}$ PBS (Lee et al., 2013) and coverslipped with Hardset Mounting Medium (Vector Labs). Images of VGAT-Venus expression in barreloids in the left hemisphere were captured using a Leica TCS SP2 confocal microscope (Leica Microsystems, Wetzlar, Germany) or a Retiga-EX camera mounted on an Olympus BX-51 upright microscope (Olympus, Shinjuku, Tokyo, Japan). Contrast of digitized images was enhanced in Fiji (Schindelin et al., 2012; biological image software based on ImageJ, National Institutes of Health, Bethesda, MD, USA) using same parameters.

\section{Vessicular Glutamate Transporter 2 (VGLUT2) Immunohistochemistry}

For vessicular glutamate transporter 2 (VGLUT2) staining, sections were blocked with $10 \%$ normal goat serum (Vector Laboratories) and $0.5 \%$ Triton X-100 in PBS for $30 \mathrm{~min}$, and incubated with 1:5,000 guinea pig anti-VGLUT2 antibody (Millipore, Bedford, MA, USA) overnight and 1:400 Alexa 568 conjugated goat anti-guinea pig IgG (Life Technologies, Carlsbad, CA, USA) for $2 \mathrm{~h}$. Counterstaining was performed for nuclear staining using 1-2 $\mu \mathrm{M}$ To-Pro-3 Iodide (Life Technologies, Carlsbad, CA, USA) for $15 \mathrm{~min}$. These sections were covered with Hardset Mounting Medium (Vector Laboratories, Burlingame, CA, USA) and imaged as described above.

\section{Dil Deposit}

Brains were removed, blocked and fixed at $\mathrm{P} 3$, as described above. The blocked brains were mounted on a stage with instant glue adhesive and submerged in $10 \mathrm{mM}$ PBS. Then, 200-300 $\mu \mathrm{m}$ sections were collected in $10 \mathrm{mM}$ PBS using a vibratome (Ted Pella, Redding, CA, USA). Selected sections containing the TRN and the thalamic barreloid border (e.g., Figure 5A) were further fixed in $4 \%$ fresh PFA/PBS overnight. To estimate whether RT fibers were present at $\mathrm{P} 3$ in this slice preparation, small crystals of DiI (Life Technologies, Carlsbad, CA, USA) were manually deposited on the TRN in our slice preparations using an insect pin visually guided under a dissecting microscope (AmScope, Irvine, CA, USA). DiI enables fiber tracing in postmortem, fixed tissue (Chua et al., 1990; Ozaki and Wahlsten, 1992). These sections were incubated at $37^{\circ} \mathrm{C}$ in $4 \% \mathrm{PFA} / \mathrm{PBS}$ for a month 
and mounted on a slide using spacers (Electron Microscopy Sciences, Hatfield, PA, USA) and Fluoro-Gel mounting medium (Electron Microscopy Sciences) after washing in PBS. Images were captured by an Olympus Fluoview FV1000 confocal microscope (Olympus).

\section{Whisker Lesion}

Pups were anesthetized by inhalation of isoflurane (1\%-5\%). Before the surgery, meloxicam $(1 \mathrm{mg} / \mathrm{kg})$ was injected subcutaneously. Under a dissection microscope, whiskers in $\mathrm{C1}-\mathrm{C3}$ on the right side of the snout were carefully plucked out using a pair of sharp tweezers (Takeuchi et al., 2014), and these whisker follicles were surgically removed using a pair of sharp tweezers after making small incisions along the $C$ barreloid row at $\mathrm{P} 2-\mathrm{P} 3$ or $\mathrm{P} 5$, respectively. Whiskers in these animals were observed to confirm no whisker growth before sacrificing animals. At P10, these animals were deeply anesthetized under isoflurane and fixed by $4 \%$ PFA/PBS. The brains were collected, postfixed, cryoprotected, sectioned, processed and imaged, as described above.

\section{Analysis}

For offline analysis of electrophysiological recording locations, we identified each barreloid based on the position of the dorsal edge of the TRN (illustrated by an arrow head in Figures 3, 4), which is usually aligned with $B$ barreloid row. However, four recording locations could not be unambiguously identified due to technical problems with our alignment. The inhibitory input field (IIF) was defined as the region of the TRN that elicited responses from the recorded VB neuron. This was estimated as pixel numbers using Fiji as follows:

$$
\frac{\text { overlapped IIF (pixels) }}{\text { sum of two IIFs (pixels) }} \times 100(\%)
$$

Distance between locations in the barreloids was estimated as pixel numbers in a linear measure using Fiji (Schindelin et al., 2012). To understand the relationship between overlapped IIFs in the TRN and the distance of recording locations in barreloids, we performed a non-linear regression analysis using an exponential decay model (Figure 3D). To assess topographic organization between IIFs in the TRN and recording locations in barreloids, IIF centers and recording locations were collapsed along an abscissa (Figure 3E) or an ordinate (Figure 3F), respectively, and a linear regression analysis was performed separately.

To quantify development of inhibitory projections to the barreloids, we introduce a barreloid development index (BDI). VGAT-Venus labeled barreloids and VGLUT2 labeled barreloids were scored by identifying shared barreloids. The BDI indicates when barreloids were labeled by both VGAT and VGLUT2:

$$
\begin{aligned}
& \text { the number of VGAT barreloids } \\
& \text { the number of VGLUT2 barreloids }
\end{aligned}
$$

BDI indices of zero indicate that no VGAT-labeled barreloids are found, while indicies near one indicate complete overlap of VGAT-labeled and VGLUT2-labeled barreloids. We quantified BDI in two to three sections from each brain, and the mean
BDI score from each brain was plotted in Figure 4D. Complete barreloids appear only in a few sections at P8 or older animals. Raw data from all experiments are freely available for sharing upon request.

\section{RESULTS}

A major problem with studying barreloids in the somatosensory thalamus is the technical difficulty with accurately and unambiguously delineating the barreloid architecture. To solve this problem, we developed a slice preparation (Figure 2) that enables the straightforward identification of each barreloid. Our preparation takes advantage of transgenic-labeling of VGAT-positive inhibitory neurons, i.e., the VGAT-Venus mouse line (Wang et al., 2009). VGAT-Venus mice have been widely used to identify inhibitory neurons with Venus-labeled neuronal somata in various studies (Inada et al., 2011; Arami et al., 2013; Henderson et al., 2014; Bolton et al., 2015; Lee et al., 2015a,b). In addition, Venus is expressed in inhibitory axonal projections (Saito et al., 2015). We fully utilized these Venuslabeled projections from the TRN to understand synaptic and structural properties of the RT inhibitory projections. The structure of thalamic barreloids in a fixed slice is unambiguously delineated by Venus labeled RT projections from the TRN, since there are practically no intrinsic local inhibitory neurons in the rodent somatosensory thalamus (Cox et al., 1996; Guillery and Harting, 2003; Figure 3A). Using this preparation, each barreloid can also be easily identified in a live brain slice, for targeted physiological recordings from specific barreloids (Figure 3B).

\section{Structural Basis of Topographic Projections}

To physiologically map the RT projections to specific barreloids, we made whole-cell recordings from barreloid neurons in an in vitro brain slice preparation. We confirmed the recording locations in each barreloid by offline examinations of the recording pipette placement. The inhibitory projections of the TRN were assessed by uncaging glutamate using laser-scanning photostimulation while making whole-cell recordings from a neuron in an identified barreloid (Lee et al., 2013). To isolate inhibitory currents, the resting membrane potentials of neurons were held at $0 \mathrm{mV}$ using a cesium intracellular solution. Recorded barreloid neurons exhibited IPSCs responding to photostimulation of neurons within the TRN (Figure 3B inset). For all recorded barreloid neurons, the projection origin in the TRN was localized to $1-2$ stimulation spots ( $80 \mu \mathrm{m}$ separation between adjacent stimulation spots; Figure 3B inset). These stimulation loci were generally elongated along the long-axis of the TRN. Thus, these 1-2 activated stimulation spots in the TRN constitute the inhibitory TRN IIF of each barreloid neuron (see "Materials and Methods" section).

As an example, four recorded cells in B1, C3, D3 and E4 barreloids from the same live slice preparation received inhibitory RT input from topographically segregated but overlapped IIFs (5\%-17\%) in TRN (Figure 3C). However, no overlapped IIF was found when the recording locations 
A

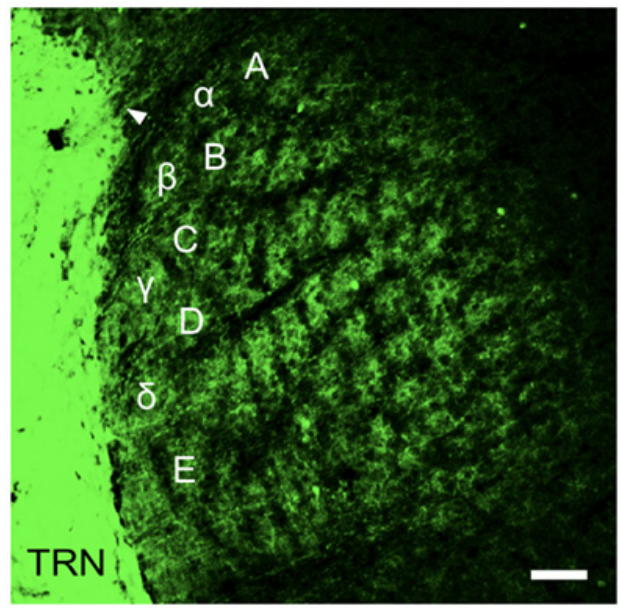

C
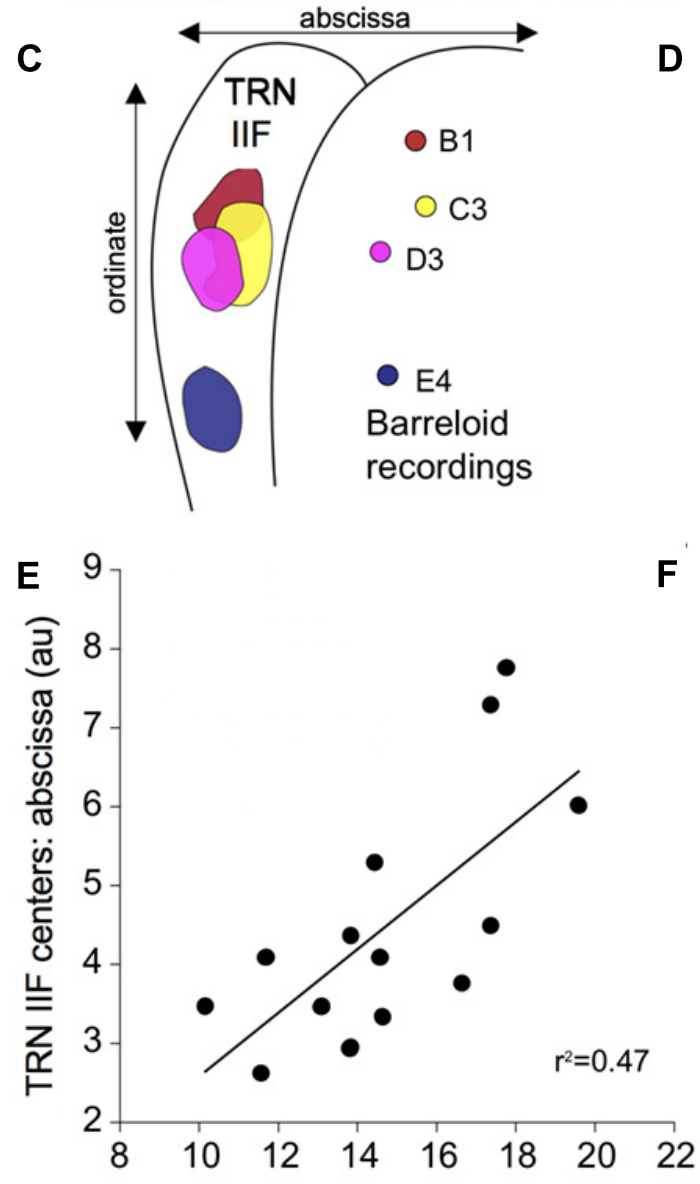

Barreloid recordings: abscissa (au)

D
B
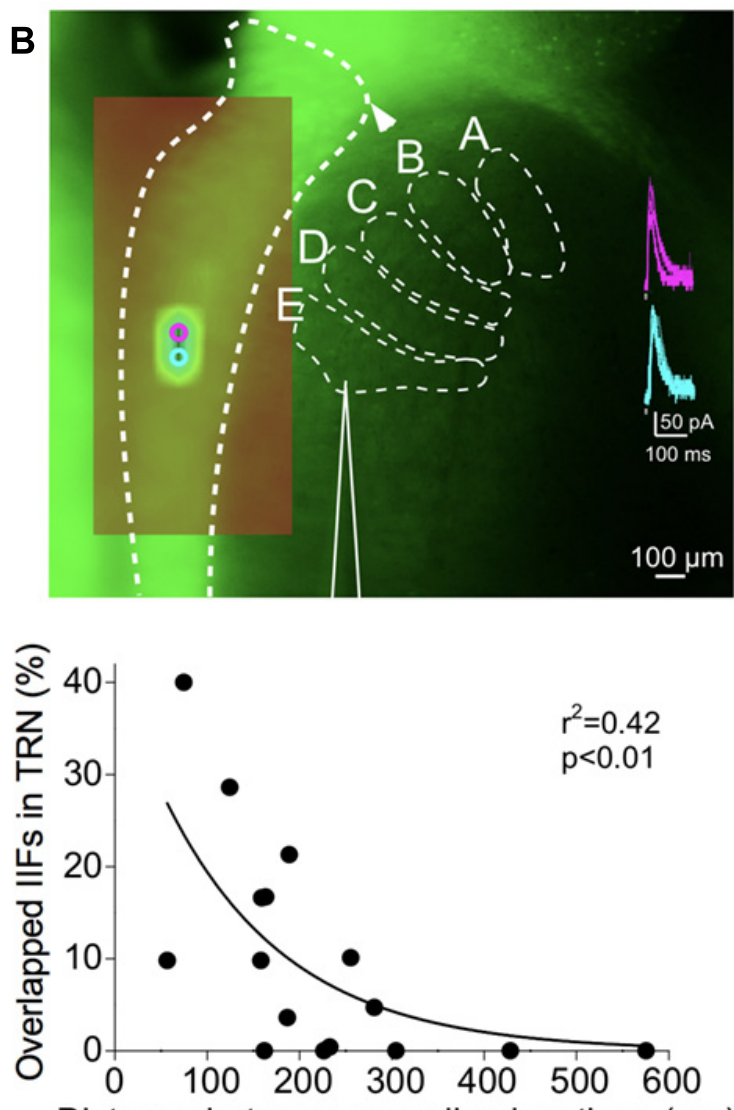

Distance between recording locations $(\mu \mathrm{m})$

F

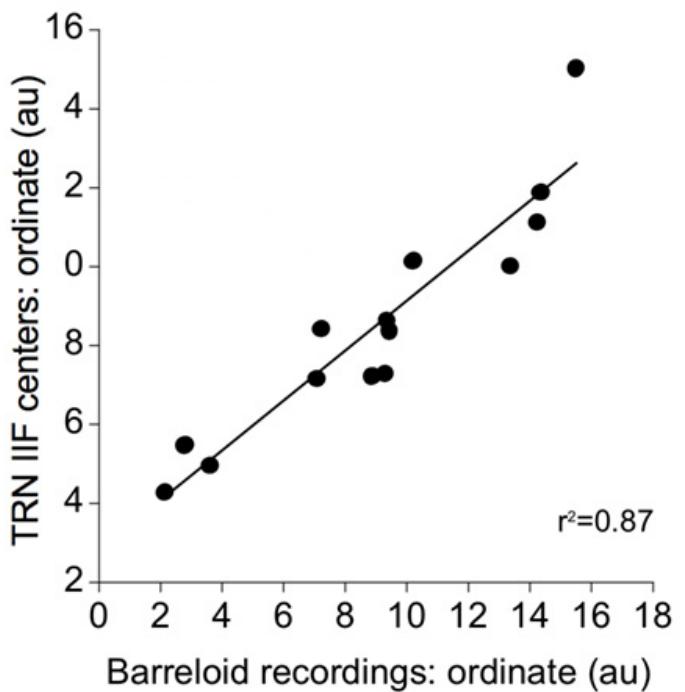

FIGURE 3 | Functional topography of inhibitory projections to the barreloids. (A) Confocal image of inhibitory projections to the barreloids in a fixed brain slice $(50 \mu \mathrm{m}$ thickness) at P12. Arrowhead indicates alignment of the edge of the TRN with the $B$ barreloid row. Scale: $100 \mu \mathrm{m}$. (B) Inhibitory projections to the barreloids as observed in the live in vitro brain slice (500 $\mu \mathrm{m}$ thickness). (A-C) Illustrates barreloid rows for reference. For identifying barreloids, the dorsal edge of the TRN (illustrated by the arrowhead) is a landmark, which is often aligned with the $B$ barreloid row. Scale: $100 \mu \mathrm{m}$. (inset) Representative inhibitory postsynaptic currents elicited by photostimulation of the TRN. (C) Four representative inhibitory input fields (IIFs) in the TRN and their corresponding recording locations in thalamic barreloids from a same slice. Overlapped IIFs in TRN are 17\% (B1-C3), 5\% (B1-D3), 17\% (C3-D3) and 0\% with E4. (D) Significant relationship between overlapped IIFs in the TRN and distance between recording locations in thalamic barreloids. Sixteen recording pairs from the same slices $(n=4)$ were analyzed using non-linear regression. (E) Topographic organization between IIFs in TRN and recording locations in thalamic barreloids along the abscissa. Fourteen IIFs and the corresponding recording locations from all recorded neurons are aligned on a single map. IIF centers in TRN and recording locations in barreloids are significantly correlated in a linear regression. (F) Same as $\mathbf{( E )}$ except for analysis along the ordinate. 

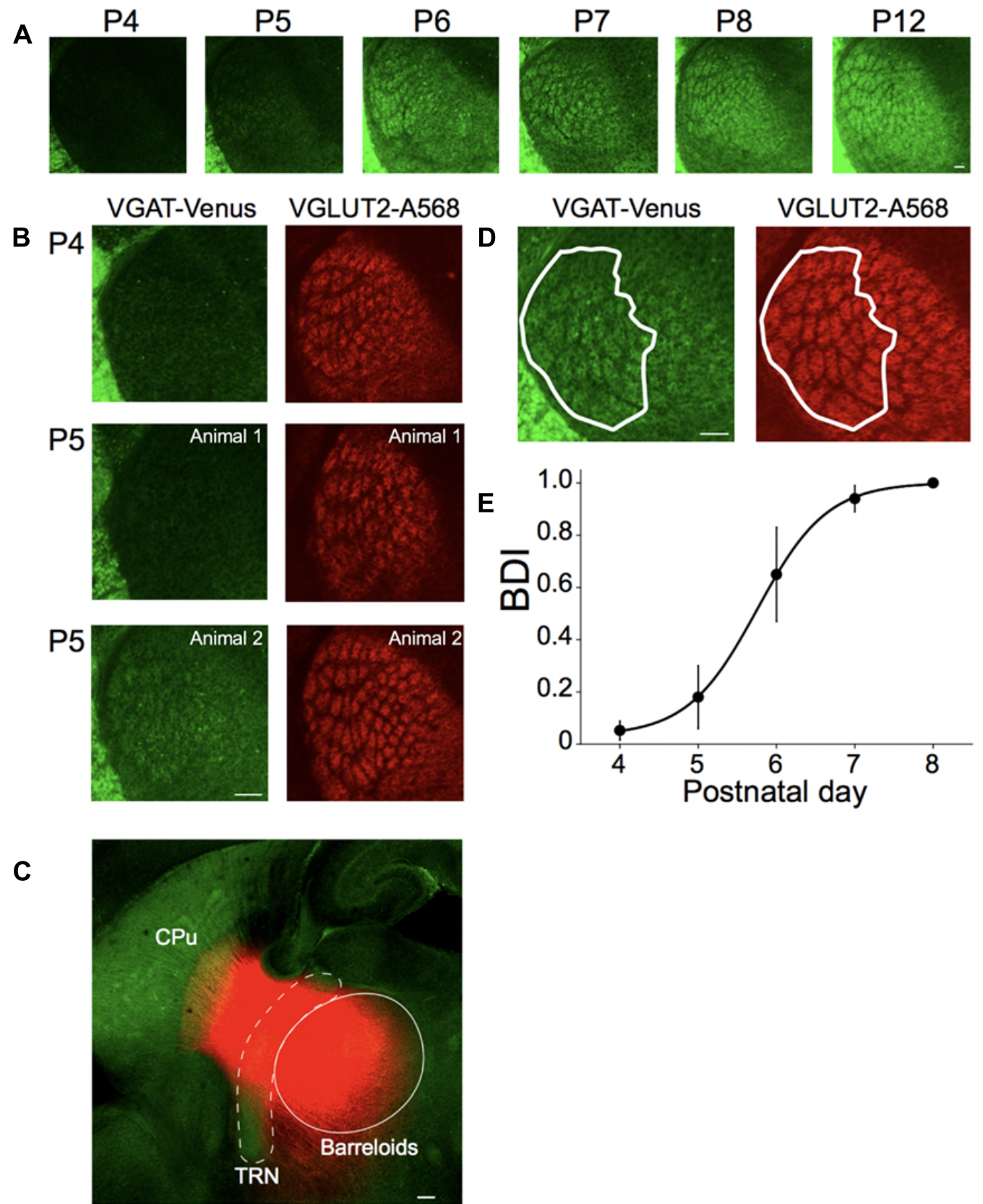

FIGURE 4 | Postnatal development of inhibitory projections to the barreloids. (A) Development of inhibitory projections to the barreloids from P4 to P12. For illustration purposes, brightness and contrast of the images are enhanced. (B) Different development of inhibitory and excitatory projections to the barreloids. Left panels: inhibitory projections to the barreloids expressed by vesicular gamma-aminobutryic acid (GABA) transporter (VGAT)-Venus. Right panels: excitatory projections to the barreloids expressed by vessicular glutamate transporter 2 (VGLUT2) with Alexa 568 (A568). At P5-6, development of inhibitory projections to the barreloids exhibit large individual variability, which is also evident in the large SEM of the barreloid development index BDI scores in (E). BDI scores: 0 (top), 0 (middle) and 0.63 (bottom). (C) Existence of reticulothalamic (RT) projections before P4. Small crystals of Dil were placed on the TRN in fixed sections (200-300 $\mu \mathrm{m}$ ) at P3 and incubated in $4 \%$ paraformaldehyde (PFA) at $37^{\circ} \mathrm{C}$ for a month. Dil Labeling show an exponential decrease in intensity in the thalamus and striatum, likely representing the edge of transport for the dye, or the termination of these fibers in this slice preparation. In addition, since the borders of the barreloids observed using VGAT are not discernable at P3, we only indicate an approximate area for the barreloids. More precise delineation with VGLUT2 staining was not feasible in the thick slice prior to Dil labeling. The approximate TRN and barreloid regions are illustrated by white broken line and white line, respectively. RT projections are red. $\mathrm{CPu}$, caudate putamen. (D) Identification of inhibitory projections to the barreloids (VGAT-Venus) shared with excitatory projections to the projections to the barreloids (VGLUT2-A568). A white line illustrates barreloid border analyzed for BDI. BDI score: 1. This section was obtained from the brain at P7. (E) Establishment of inhibitory projections to the barreloids. BDI scores are plotted as a function of postnatal day (mean \pm SEM). Sigmoidal fitting reaches a plateau at P7-P8, indicating that inhibitory projections to the barreloids are established by P7-8. Number of animals: $n=3$ (P4), $n=5$ (P5), $n=3$ (P6), $n=6$ (P7) and $n=3$ (P8). Scale bars in each panel: $100 \mu \mathrm{m}$ (A-D). 
were sufficiently separated, such as in three barreloids and the E4 barreloid (Figure 3C). Overlapped IIFs in the TRN vary from $0 \%$ to $40 \%$, which are significantly and inversely correlated with distance between recording locations: the shorter the distance between recording locations in VGAT-labeled barreloids, the more the IIFs overlapped in the TRN (Figure 3D). We decomposed 14 TRN IIFs in a single map. Similar to that previously reported for projections from the TRN and somatosensory thalamus (Lam and Sherman, 2005, 2011; Lam et al., 2006), we found a topographic organization between IIF centers in the TRN and recording locations in thalamic barreloids (Figures 3E,F).

\section{Development of Inhibitory Projections to the Barreloids}

Brainstem barrelettes, thalamic barreloids and cortical barrels are defined by functional clusters of ascending excitatory axons. We distinguished the VGAT-Venus labeled barreloids formed by inhibitory RT projections from those formed by feedforward excitatory projections using immunohistochemical labeling for VGLUT2, which is expressed on the ascending excitatory terminals.

The excitatory projections to the barrelette, barreloid and barrel structures are consecutively established by postnatal day 0-1 (P0-P1), P2-P3 and P3-P5, respectively, which follows the arrival of ascending excitatory axons from the lower to the higher station by embryonic day 12 (E12), E17, and P0, respectively (Ma, 1993; Erzurumlu and Gaspar, 2012; Mizuno et al., 2014; Takeuchi et al., 2014; Yamasaki et al., 2014; Figure 1). However, inhibitory projections to the barreloids are formed by feedback inhibitory RT axons from the TRN that receives collateral excitatory CT input from layer 6 of the barrel cortex (Figure 1). Ascending excitatory axons from the brainstem developmentally reach the thalamus by E17 (Erzurumlu and Gaspar, 2012), whereas descending $\mathrm{CT}$ as well as $\mathrm{RT}$ axons reach the thalamus at E18-E19 (Mitrofanis and Baker, 1993; Jacobs et al., 2007; Grant et al., 2012; Figure 1). It is also generally accepted that inhibitory circuits in the central sensory system developmentally lag behind excitatory circuits (Chang et al., 2005; Tao and Poo, 2005; Dorrn et al., 2010). Thus, we hypothesized that development of the inhibitory projections to the barreloids also lags behind that of the excitatory projections to the barreloids.

To test this hypothesis, first, we examined the development of inhibitory projections to the barreloids at different postnatal developmental periods (P4-P12) in fixed brain slices and quantified their development, as described below (Figure 4A). At P4, the outline of barreloids is formed, but VGAT-labeled barreloids are not yet visible. At P5-P6, individual VGAT-labeled barreloids are more visible. By P7-P8, VGAT-labeled barreloids clearly separated by septa are recognizable. At P12, the fully formed structure of the VGAT-labeled barreloids is clearly evident.

To confirm this developmental delay for the establishment of inhibitory projections to the barreloids compared to excitatory projections to the barreloids, we immunohistochemically stained for VGLUT2 in the developing brain at P4-P8. VGLUT2 is expressed in the ascending excitatory axonal projections from the brainstem (Kivrak and Erzurumlu, 2013). Whereas excitatory projections to the barreloids are established by P4 (Figures 1, 4B), inhibitory projections to the barreloids are not yet fully developed at P4 and exhibits individual variability at P5 during development (Figure 4B). Previous studies in the rat have reported that RT projections already exist before birth, but are immature and not fully functional until the second postnatal week (De Biasi et al., 1996, 1997; Figure 1). However, it is not clear whether RT projections also exist in the mouse even at $\mathrm{P} 4$ from our preparations. We investigated the existence of RT projections before $\mathrm{P} 4$ (before the appearance of VGAT-labeled barreloids). We placed a fluorescent lipophilic indocarbocyanine orange-red dye, DiI, in the TRN in fixed brain slices at P3 and found labeled fibers that emanated from the deposit site toward the region of VB and the striatum (Figure 4C). These fibers were consistent with the suggested early presence of RT projections from prior studies (De Biasi et al., 1996, 1997), although it is also likely that labeling of TC and/or CT fibers also contributed to the observed labeling.

To quantify the different developmental time course of excitatory and inhibitory projections to the barreloids, we identified the number of VGAT-labeled barreloids shared with VGLUT2-labeled barreloids at P4-P8 (Figure 4D). BDI (see "Materials and Methods" section) varies from zero (a ratio between identical inhibitory and excitatory projections to the barreloids) indicating no developed inhibitory projections to the barreloid to one, indicating a full establishment of inhibitory projections to the barreloids. A sigmoidal fitting of BDI scores reaches a plateau by P7-P8 (Figure 4E), suggesting that inhibitory projections to the barreloids are established by P7-P8 and lag 5 days behind excitatory projections to the barreloids. This is not an artifact caused by slow expression of VGAT-Venus because VGAT-Venus is already expressed in cell bodies as well as neurites at P0 (Inada et al., 2011).

\section{Critical Period of Structural Plasticity in Inhibitory Projections to the Barreloids}

We next sought to understand structural plasticity of the inhibitory projections to the barreloids. It has been well documented that the critical period of structural plasticity of the thalamic excitatory projections to the barreloids and the barrel cortex closes by P4 (Yamakado, 1999; Erzurumlu and Gaspar, 2012). This period is after the establishment of excitatory projections to the barreloids (P2-P3) and during the establishment of the barrel cortex (P3-P5; Figure 1). Given the slow development of inhibitory projections to the barreloids (P7-P8; Figure 4), the critical period of structural plasticity in the inhibitory projections to the barreloids might extend beyond $\mathrm{P} 4$, similar to that found in the visual system, where the critical period of inhibitory neurons in ocular dominance plasticity of the mouse primary visual cortex lags behind that of excitatory neurons (Gandhi et al., 2008).

Here, we lesioned whisker follicles in the $\mathrm{C}$ row $(\mathrm{C} 1, \mathrm{C} 2$ and C3) on the right side of the snout at P2-3 or P5, respectively, in separate animals. We assessed the outcome of barreloid structure following whisker lesions by examining the barreloid architecture at P10. When the whiskers were lesioned at P3, the 


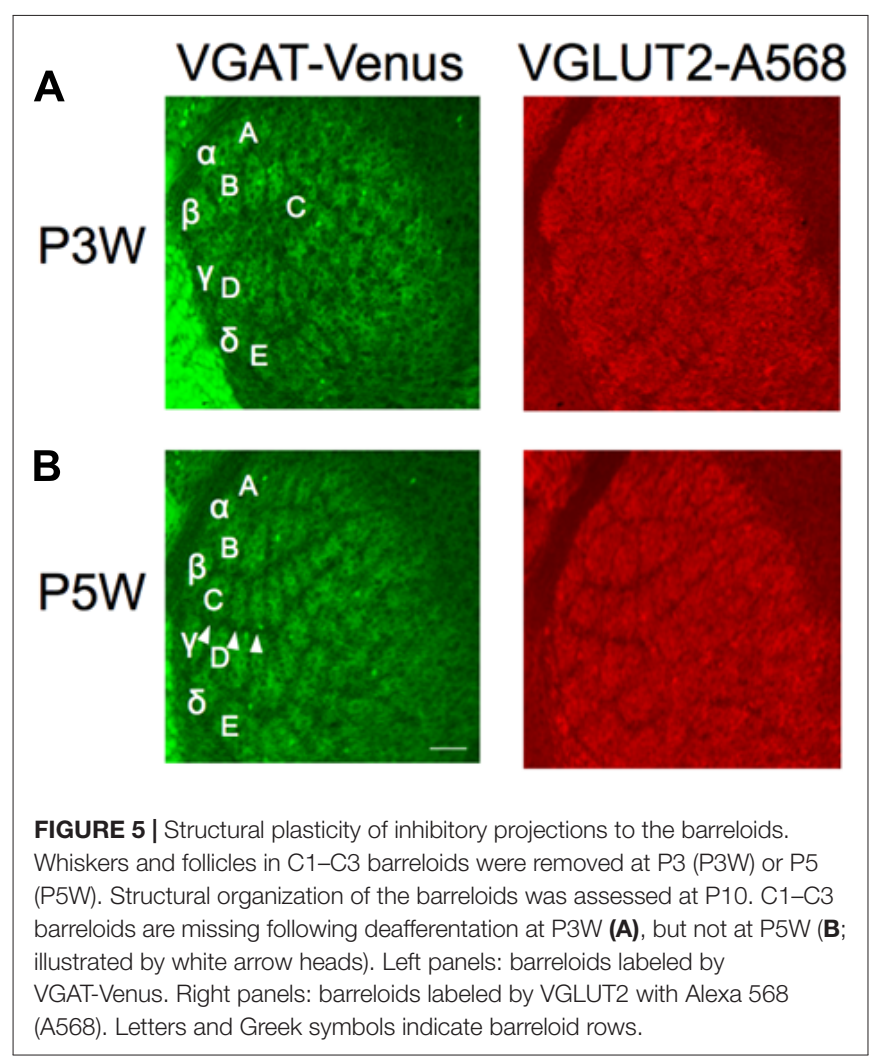

original thalamic area of the excitatory and inhibitory inputs to $\mathrm{C} 1-\mathrm{C} 3$ barreloids was replaced by D barreloids (Figure 5A). We then tested whether similar structural plasticity occurs by the same whisker lesions at P5. Based on our finding that the developmental period of inhibitory projections to the barreloids lagged behind that of the excitatory projections (Figure 4D), we posited that the critical period for the inhibitory projections might also lag behind that of the excitatory projections. However, we found that whisker lesions at P5 did not result in structural plasticity for either the excitatory or inhibitory projections to the barreloids (Figure 5B). Thus, we found a similar critical period prior to P5 for both the excitatory and inhibitory projections to the barreloids.

\section{DISCUSSION}

In this study, we developed a preparation that preserves inhibitory projections to the barreloids in a live slice for investigating functional topography. Unlike the barrel cortex, the barreloids are much less appreciated as a model system due to the difficulty of delineation. However, by using VGAT-Venus transgenic mice, we were able to capitalize on the rapid and unambiguous visualization of barreloid architecture in both live and postmortem slices.

\section{Barreloid Basis of Topographic Organization}

Feedback RT projections are topographically organized (Lam and Sherman, 2005, 2011; Lam et al., 2006). Since our preparations allowed us to identify each barreloid online using epifluorescent microscopy, we extended previous studies of $\mathrm{RT}$ architecture that were unable to assign recording location to specific barreloids (Lam et al., 2006). We found that different barreloids have overlapped IIFs in TRN, potentially explained by the previous finding that individual TRN neurons have axon terminal bundles in more than one barreloid (Cox et al., 1996). Thus, these divergent projections from the TRN match the convergent projections to the barreloids in our results. The topography of RT barreloid projections was well preserved from recordings in single sections. However, when data were combined from separate experiments, this barreloid projection topography was less (yet highly significant statistically) preserved, perhaps due to different barreloid size and alignment artifacts across different animals. In the current study, we made recordings from neurons in up to four separate barreloids in a single slice preparation.

Future studies can utilize this preparation to understand the topographic organization within a single barreloid as well as along a single barreloid row or column. Moreover, the ability to identify and target specific barreloids for in vitro recordings can greatly simplify future studies of whisker sensation that combine slice physiology with in vivo experimentation.

\section{Delayed Development of Inhibitory Projection to the Barreloids}

A surprising finding from our study is the delayed development of inhibitory projections to the barreloids compared to that of excitatory projections to the barreloids. The barrel system (e.g., the excitatory projections to the barreloids and the barrel cortex) is formed a few days after the arrival of ascending axons (Figure 1; Erzurumlu and Gaspar, 2012). Both excitatory axons from the brainstem and inhibitory axons from the TRN arrive at the thalamus before birth (E17 for excitatory axons and E18-E19 for inhibitory axons; Erzurumlu and Gaspar, 2012; Grant et al., 2012). Whereas the excitatory projections to the barreloids are formed at P2-P3, the inhibitory projections to the barreloids are formed at P7-P8, which lags 5 days behind that of the excitatory projections to the barreloids. This delay in the formation of the inhibitory projections to the barreloids cannot be simply accounted for by different axonal arrival time (Figure 1; Erzurumlu and Gaspar, 2012).

Development and maturation of inhibitory circuits in sensory systems lags behind that of the excitatory circuits (Chang et al., 2005; Tao and Poo, 2005; Dorrn et al., 2010). Indeed, prior ultrastructural studies of the developing RT projection to VB indicate that GABAergic synaptic terminals are present, but immature at birth and only reach full maturity by the second postnatal week in rats (De Biasi et al., 1996, 1997). Therefore, additional mechanisms likely account for delayed development of the inhibitory projections to the barreloids. One possibility is feedback excitatory input from cortical layer 6. Layer $6 \mathrm{CT}$ projections reach the barreloids as early as E18 (Jacobs et al., 2007), but take longer to fully innervate the barreloids between P2 and P6 (Grant et al., 
2012). The later time of this period (P5-P6) roughly matches the developmental period of the inhibitory projections to the barreloids. Perhaps, during this period, a balance between excitation and inhibitory inputs can be established for developing feedback projections (Froemke, 2015). Interestingly though, cortical layer 6 receives direct TC projections (Wimmer et al., 2010; Lee et al., 2012; Lee and Imaizumi, 2013), which form barrel-like fields in layer 6a, termed infrabarrels (Crandall et al., 2017). In the future, it will be necessary to examine how these infrabarrel layer 6 neurons affect development and formation of the inhibitory projections to the barreloids.

\section{Fixed Critical Period of Structural Plasticity in Inhibitory Projections to the Barreloids}

Another surprising finding from this study is the fixed critical period of structural plasticity in the inhibitory projections to the barreloids. It has been well documented that the critical period of structural plasticity in the excitatory projections to the barreloids closes by P4 (Yamakado, 1999; Erzurumlu and Gaspar, 2012). In our study, whisker lesions before P4 resulted in structural plasticity both in excitatory and inhibitory projections to the barreloids (Figure 5). The same procedure was performed at P5 after the closure of the excitatory critical period but still during the development period of inhibitory projections to the barreloids. We found that the critical period of structural plasticity in the inhibitory projections to the barreloids had already closed despite the delayed development of the inhibitory projections to the barreloids. This suggests that the critical period of structural plasticity closes at the same time for both the excitatory and inhibitory projections to the barreloids and that delayed development of the inhibitory projections to the barreloids does not affect timing of critical period closure.

The molecular mechanisms underlying the critical period changes appear to be in part dependent on NMDA receptors, in particular NR2B, which affects barreloid development and the critical period closure of structural plasticity: early or delayed development corresponds to early or late closure of the critical period, respectively (Yamasaki et al., 2014). However, other NMDA receptors, NR2A and NR2D appear less important in this regard (Lu et al., 2001). It remains to be determined whether analogous effects are mediated through different GABA receptor subtypes, or other neurotransmitter receptors. These receptors are known to exhibit a developmental switch in the immature thalamus (Peden et al., 2008).

Physiologically, RT projections are able to elicit relatively weak responses at ages up through P5, whereas afterwards the strength of inhibitory responses increases dramatically (Evrad and Ropert, 2009). Thus, it is likely, as suggested from prior ultrastructural studies, that weak inhibitory RT to VB barreloid connections are present to be refined through signaling in the same critical window (De Biasi et al., 1996, 1997). Alternatively, as a group, thalamic reticular neurons are composed of chemically distinct subtypes, among which several express the calcium binding protein, calretinin (Lizier et al.,
1997), which has also been shown to be poorly labeled in VGAT-Venus mice (Uematsu et al., 2008). Thus, a subset of RT projections may be present earlier, but not revealed in our VGAT-Venus preparation.

Potential insight into the formation of inhibitory projections to the projections to the barreloids may be drawn from related studies of the critical period of ocular dominance plasticity in the primary visual cortex, where a number of neural mechanisms underlying ocular dominance plasticity have been proposed (Hensch, 2005; Levelt and Hübener, 2012). Local inhibitory neurons may undergo structural and synaptic refinement, largely to regulate excitatory synaptic inputs. More recent studies have also proposed a contribution of microglia to synaptic pruning of excitatory projections (Schafer et al., 2012, 2013). Overall though, the focus is generally on the development of excitatory projections. And, it remains to understand whether similar synaptic pruning mechanisms underlie development, structural plasticity, and critical period of inhibitory projections.

\section{Perspectives}

Our study raises several questions regarding the inhibitory projections to the barreloids that can be addressed using our slice preparation in the VGAT-Venus transgenic mouse line: canonical topographic organization, effects of cortical layer 6 on the development of inhibitory projection to the barreloids, and temporal pattern of synaptic pruning on inhibitory projections. In the future, it is also possible to observe excitatory and inhibitory synaptic projection-interactions with excitatory axons (e.g., from the brainstem or cortical layer 6) by crossing with relevant transgenic mouse lines. Such detailed studies should shed light not only on the development and structural plasticity of the barreloids but also their relevance to the neural mechanisms underlying related psychiatric disorders.

\section{AUTHOR CONTRIBUTIONS}

KI designed the experiments. KI and CL conducted the experiments and analyzed the data. YY developed and provided the VGAT-Venus mouse line. KI, GF and CL drafted and edited the manuscript.

\section{FUNDING}

This study was supported by National Institutes of Health (NIH) grants R03 AG 052120 and R03 MH11361 and NSF Grant IOS 1652432 (to CL) and SVM CORP grant LAV 3487 (to KI), and by Stanley Center for Psychiatric Research at Broad Institute of MIT and Harvard (to GF).

\section{ACKNOWLEDGMENTS}

We thank Sherry Ring for her histological assistance, Alberto Musto for his technical instruction, and Violeta Gisselle Lopez Huerta and Tobias Keiser for preliminary experiments. 


\section{REFERENCES}

Arami, M. K., Sohya, K., Sarihi, A., Jiang, B., Yanagawa, Y., and Tsumoto, T. (2013). Reciprocal Homosynaptic and heterosynaptic long-term plasticity of corticogeniculate projection neurons in layer VI of the mouse visual cortex. J. Neurosci. 33, 7787-7798. doi: 10.1523/JNEUROSCI.5350-12.2013

Bolton, A. D., Murata, Y., Kirchner, R., Kim, S. Y., Young, A., Dang, T., et al. (2015). A diencephalic dopamine source provides input to the superior colliculus, where D1 and D2 receptors segregate to distinct functional zones. Cell Rep. 13, 1003-1015. doi: 10.1016/j.celrep.2015.09.046

Chang, E. F., Bao, S., Imaizumi, K., Schreiner, C. E., and Merzenich, M. M. (2005). Development of spectral and temporal response selectivity in the auditory cortex. Proc. Natl. Acad. Sci. U S A 102, 16460-16465. doi: 10.1073/pnas. 0508239102

Chua, C. K., Balice-Gordon, R. J., and Lichtman, J. W. (1990). Differential labeling of terminal arbors of multiple axons innervating the same target all with DiI and a new lipophilic tracer. Soc. Neurosci. Abstr. 16:1004.

Cox, C. L., Huguenard, J. R., and Prince, D. A. (1996). Heterogeneous axonal arborizations of rat thalamic reticular neurons in the ventrobasal nucleus. J. Comp. Neurol. 366, 416-430. doi: 10.1002/(SICI)10969861(19960311)366:3<416::AID-CNE4>3.0.CO;2-7

Crandall, S. R., Patrick, S. L., Cruickshanks, S. J., and Connors, B. W. (2017). Infrabarrels are layer 6 circuit modules in the barrel cortex that link long-range inputs and outputs. Cell Rep. 21, P3065-P3078. doi: 10.1016/j.celrep.2017. 11.049

Cruikshank, S. J., Rose, H. J., and Metherate, R. (2002). Auditory thalamocortical synaptic transmission in vitro. J. Neurophysiol. 87, 361-384. doi: 10.1152/jn. 00549.2001

De Biasi, S., Amadeo, A., Arcelli, P., Frassoni, C., Meroni, A., and Spreafico, R. (1996). Ultrastructural characterization of the postnatal development of the thalamic ventrobasal and reticular nuclei in the rat. Anat. Embryol. 193, 341-353. doi: 10.1007/bf00186691

De Biasi, S., Amadeo, A., Arcelli, P., Frassoni, C., and Spreafico, R. (1997). Postnatal development of GABA-immunoreactive terminals in the reticular and ventrobasal nuclei of the rat thalamus: a light and electron microscopic study. Neuroscience 76, 503-515. doi: 10.1016/s0306-4522(96)00376-4

Dorrn, A. L., Yuan, K., Barker, A. J., Schreiner, C. E., and Froemke, R. C. (2010). Developmental sensory experience balances cortical excitation and inhibition. Nature 465, 932-936. doi: 10.1038/nature09119

Erzurumlu, R. S., and Gaspar, P. (2012). Development and critical period plasticity of the barrel cortex. Eur. J. Neurosci. 35, 1540-1553. doi: 10.1111/j.1460-9568. 2012.08075. $\mathrm{x}$

Espinosa, J. S., and Stryker, M. P. (2012). Development and plasticity of the primary visual cortex. Neuron 75, 230-249. doi: 10.1016/j.neuron.2012.06.009

Evrad, A., and Ropert, N. (2009). Early development of the thalamic inhibitory feedback loop in the primary somatosensory system of the newborn mice. J. Neurosci. 29, 9930-9940. doi: 10.1523/JNEUROSCI.1671-09.2009

Froemke, R. C. (2015). Plasticity of cortical excitatory-inhibitory balance. Annu. Rev. Neurosci. 38, 195-219. doi: 10.1146/annurev-neuro-071714-034002

Gandhi, S. P., Yanagawa, Y., and Stryker, M. P. (2008). Delayed plasticity of inhibitory neurons in developing visual cortex. Proc. Natl. Acad. Sci. U S A 105, 16797-16802. doi: 10.1073/pnas.0806159105

Grant, E., Hoerder-Suabedissen, A., and Molnár, Z. (2012). Development of the corticothalamic projections. Front. Neurosci. 6:53. doi: 10.3389/fnins.2012. 00053

Guillery, R. W., and Harting, J. K. (2003). Structure and connections of the thalamic reticular nucleus: advancing views over half a century. J. Comp. Neurol. 463, 360-371. doi: 10.1002/cne.10738

Henderson, K. W., Gupta, J., Tagliatela, S., Litvina, E., Zheng, X., Van Zandt, M. A., et al. (2014). Long-term seizure suppression and optogenetic analyses of synaptic connectivity in epileptic mice with hippocampal grafts of GABAergic interneurons. J. Neurosci. 34, 13492-13504. doi: 10.1523/JNEUROSCI.000514.2014

Hensch, T. K. (2005). Critical period plasticity in local cortical circuits. Nat. Rev. Neurosci. 6, 877-888. doi: 10.1038/nrn 1787

Imaizumi, K., and Lee, C. C. (2014). Frequency transformation in the auditory lemniscal thalamocortical system. Front. Neural Circuits 8:75. doi: 10.3389/fncir.2014.00075
Inada, H., Watanabe, M., Uchida, T., Ishibashi, H., Wake, H., Nemoto, T., et al. (2011). GABA regulates the multidirectional tangential migration of GABAergic interneurons in living neonatal mice. PLoS One 6:e27048. doi: 10.1371/journal.pone.0027048

Inan, M., and Crair, M. C. (2007). Development of cortical maps: perspectives from the barrel cortex. Neuroscientist 13, 49-61. doi: 10.1177/10738584062 96257

Jacobs, E. C., Campagnoni, C., Kampf, K., Reyes, S. D., Kalra, V., Handley, V., et al. (2007). Visualization of corticofugal projections during early cortical development in a tau-GFP-transgenic mouse. Eur. J. Neurosci. 25, 17-30. doi: 10.1111/j.1460-9568.2006.05258.x

Jones, E. G. (2007). The Thalamus. Cambridge, MA: Cambridge University Press.

Kivrak, B. G., and Erzurumlu, R. S. (2013). Development of the principal nucleus trigeminal lemniscal projections in the mouse. J. Comp. Neurol. 521, 299-311. doi: $10.1002 /$ cne. 23183

Lam, Y. W., Nelson, C. S., and Sherman, S. M. (2006). Mapping of the functional interconnections between thalamic reticular neurons using photostimulation. J. Neurophysiol. 96, 2593-2600. doi: 10.1152/jn.00555.2006

Lam, Y. W., and Sherman, S. M. (2005). Mapping by laser photostimulation of connections between the thalamic reticular and ventral posterior lateral nuclei in the rat. J. Neurophysiol. 94, 2472-2483. doi: 10.1152/jn.00206.2005

Lam, Y. W., and Sherman, S. M. (2011). Functional organization of the thalamic input to the thalamic reticular nucleus. J. Neurosci. 31, 6791-6799. doi: 10.1523/JNEUROSCI.3073-10.2011

Lee, C. C., and Imaizumi, K. (2013). Functional convergence of thalamic and intrinsic projections to cortical layers 4 and 6. Neurophysiology 45, 396-406. doi: 10.1007/s11062-013-9385-2

Lee, C. C., Lam, Y. W., Imaizumi, K., and Sherman, S. M. (2013). Laser-scanning photostimulation of optogenetically targeted forebrain circuits. J. Vis. Exp. 82:50915. doi: 10.3791/50915

Lee, C. C., Lam, Y. W. and Sherman, S. M. (2012). Intracortical convergence of layer 6 neurons. Neuroreport 23, 736-740. doi: 10.1097/WNR.0b013 e328356claa

Lee, C. C., and Sherman, S. M. (2008). Synaptic properties of thalamic and intracortical intputs to layer 4 of the first- and higher-order cortical areas in the auditory and somatosensory systems. J. Neurophysiol. 100, 317-326. doi: 10.1152/jn.90391.2008

Lee, C. C., Yanagawa, Y., and Imaizumi, K. (2015a). Commissural functional topography of the inferior colliculus assessed in vitro. Hear. Res. 328, 94-101. doi: 10.1016/j.heares.2015.08.011

Lee, C. C., Yanagawa, Y., and Imaizumi, K. (2015b). Nicotinic alteration of functional thalamocortical topography. Neuroreport 26, 688-694. doi: 10.1097/wnr.0000000000000409

Levelt, C. N., and Hübener, M. (2012). Critical-period plasticity in the visual cortex. Annu. Rev. Neurosci. 35, 309-330. doi: 10.1146/annurev-neuro-061010113813

Lizier, C., Spreafico, R., and Battaglia, G. (1997). Calretinin in the thalamic reticular nucleus of the rat: distribution and relationship with ipsilateral and contralateral efferents. J. Comp. Neurol. 377, 217-233. doi: 10.1002/(sici)10969861(19970113)377:2<217::aid-cne5>3.0.co;2-6

Lokmane, L., and Garel, S. (2014). Map transfer from the thalamus to the neocortex: inputs from the barrel field. Semin. Cell Dev. Biol. 35, 147-155. doi: 10.1016/j.semcdb.2014.07.005

Lu, H. C., Gonzalez, E., and Crair, M. C. (2001). Barrel cortex critical period plasticity is independent of changes in NMDA receptor subunit composition. Neuron 32, 619-634. doi: 10.1016/s0896-6273(01)00501-3

Ma, P. M. (1993). Barrelettes-architectonic vibrissal representations in the brainstem trigeminal complex of the mouse. II. Normal post-natal development. J. Comp. Neurol. 327, 376-397. doi: 10.1002/cne.9032 70306

Mitrofanis, J., and Baker, G. E. (1993). Development of the thalamic reticular and perireticular nuclei in rats and their relationship to the course of growing corticofugal and corticopetal axons. J. Comp. Neurol. 338, 575-587. doi: $10.1002 /$ cne.903380407

Mizuno, H., Luo, W., Tarusawa, E., Saito, Y. M., Sato, T., Yoshimura, Y., et al. (2014). NMDAR-regulated dynamics of layer 4 neuronal dendrites during thalamocortical reorganization in neonates. Neuron 82, 365-379. doi: 10.1016/j. neuron.2014.02.026 
Nagai, T., Ibata, K., Park, E. S., Kubota, M., Mikoshiba, K., and Miyawaki, A. (2002). A variant of yellow fluorescent protein with fast and efficient maturation for cell-biological applications. Nat. Biotechnol. 20, 87-90. doi: $10.1038 /$ nbt0102-87

Ozaki, H. S., and Wahlsten, D. (1992). Prenatal formation of the normal mouse corpus callosum: a quantitative study with carbocyanine dyes. J. Comp. Neurol. 323, 81-90. doi: 10.1002/cne.903230107

Peden, D. R., Petitjean, C. M., Herd, M. B., Durakoglugil, M. S., Rosahl, T. W., Wafford, K., et al. (2008). Developmental maturation of synaptic and extrasynaptic GABAA receptors in mouse thalamic ventrobasal neurones. J. Physiol. 586, 965-987. doi: 10.1113/jphysiol.2007.145375

Pinault, D. (2004). The thalamic reticular nucleus: structure, function and concept. Brain Res. Rev. 46, 1-31. doi: 10.1016/j.brainresrev.2004. 04.008

Saito, Y., Zhang, Y., and Yanagawa, Y. (2015). Electrophysiological and morphological properties of neurons in the prepositus hypoglossi nucleus that express both ChAT and VGAT in a double-transgenic rat model. Eur. J. Neurosci. 41, 1036-1048. doi: 10.1111/ejn.12878

Schafer, D. P., Lehrman, E. K., Kautzman, A. G., Koyama, R., Mardinly, A. R., Yamasaki, R., et al. (2012). Microglia sculpt postnatal neural circuits in an activity and complement-dependent manner. Neuron 74, 691-705. doi: 10.1016/j.neuron.2012.03.026

Schafer, D. P., Lehrman, E. K., and Stevens, B. (2013). The "quad-partite" synapse: microglia-synapse interactions in the developing and mature CNS. Glia 61, 24-36. doi: 10.1002/glia.22389

Schindelin, J., Arganda-Carreras, I., Frise, E., Kaynig, V., Longair, M., Pietzsch, T., et al. (2012). Fiji: an open-source platform for biological-image analysis. Nat. Methods 9, 676-682. doi: 10.1038/nmeth.2019

Schreiner, C. E., and Winer, J. A. (2007). Auditory cortex mapmaking: principles, projections, and plasticity. Neuron 56, 356-365. doi: 10.1016/j.neuron.2007. 10.013

Sherman, S. M. (2016). Thalamus plays a central role in ongoing cortical functioning. Nat. Neurosci. 19, 533-541. doi: 10.1038/nn.4269

Sherman, S. M., and Guillery, R. W. (2002). The role of the thalamus in the flow of information to the cortex. Philos. Trans. R. Soc. Lond. B Biol. Sci. 357, 1695-1708. doi: 10.1098/rstb.2002.1161

Sur, M., and Leamey, C. A. (2001). Development and plasticity of cortical areas and networks. Nat. Rev. Neurosci. 2, 251-262. doi: 10.1038/35067562

Suter, B. A., O’Connor, T., Iyer, V., Petreanu, L. T., Hooks, B. M., Kiritani, T., et al. (2010). Ephus: multipurpose data acquisition software for neuroscience experiments. Front. Neural Circuits 4:100. doi: 10.3389/fncir.2010.00100

Takeuchi, Y., Asano, H., Katayama, Y., Muragaki, Y., Imoto, K., and Miyata, M. (2014). Large-scale somatotopic refinement via functional synapse elimination in the sensory thalamus of developing mice. J. Neurosci. 34, 1258-1270. doi: 10.1523/JNEUROSCI.3865-13.2014

Tao, H. W., and Poo, M. M. (2005). Activity-dependent matching of excitatory and inhibitory inputs during refinement of visual receptive fields. Neuron 45 , 829-836. doi: 10.1016/j.neuron.2005.01.046

Uematsu, M., Hirai, Y., Karube, F., Ebihara, S., Kato, M., Abe, K., et al. (2008). Quantitative chemical composition of cortical GABAergic neurons revealed in transgenic venus-expressing rats. Cereb. Cortex 18, 315-330. doi: $10.1093 /$ cercor/bhm056

Van Der Loos, H. (1976). Barreloids in mouse somatosensory thalamus. Neurosci. Lett. 2, 1-6. doi: 10.1016/0304-3940(76)90036-7

Wang, Y., Kakizaki, T., Sakagami, H., Saito, K., Ebihara, S., Kato, M., et al. (2009). Fluorescent labeling of both GABAergic and glycinergic neurons in vesicular GABA transporter (VGAT)-venus transgenic mouse. Neuroscience 164, 1031-1043. doi: 10.1016/j.neuroscience.2009.09.010

Wimmer, V. C., Bruno, R. M., de Kock, C. P., Kuner, T., and Sakmann, B. (2010). Dimensions of a projection column and architecture of VPM and POm axons in rat vibrissal cortex. Cereb. Cortex 20, 2265-2276. doi: 10.1093/cercor/bhq068

Winer, J. A., Lee, C. C., Imaizumi, K., and Schreiner, C. E. (2004). "Challenges to a neuroanatomical theory of forebrain auditory plasticity," in Plasticity and Signal Representation in the Auditory System, eds J. Syka and M. M. Merzenich (New York, NY: Klüver Academic/Plenum Publishers), 99-107.

Yamakado, M. (1999). Reassemblage of primary cell aggregates and modulation of subcortical connections in the thalamic relay nucleus: effects of vibrissal damage in the developing whisker-to-barrel pathway in the mouse. J. Comp. Neurol. 403, 517-533. doi: 10.1002/(sici)1096-9861(19990125)403:4<517::aidcne7>3.0.co; $2-4$

Yamasaki, M., Okada, R., Takasaki, C., Toki, S., Fukaya, M., Natsume, R., et al. (2014). Opposing role of NMDA receptor GluN2B and GluN2D in somatosensory development and maturation. J. Neurosci. 34, 11534-11548. doi: 10.1523/JNEUROSCI.1811-14.2014

Conflict of Interest Statement: The authors declare that the research was conducted in the absence of any commercial or financial relationships that could be construed as a potential conflict of interest.

Copyright (C) 2018 Imaizumi, Yanagawa, Feng and Lee. This is an open-access article distributed under the terms of the Creative Commons Attribution License (CC BY). The use, distribution or reproduction in other forums is permitted, provided the original author(s) and the copyright owner(s) are credited and that the original publication in this journal is cited, in accordance with accepted academic practice. No use, distribution or reproduction is permitted which does not comply with these terms. 